Check for updates

Cite this: RSC Adv., 2019, 9, 24858

Received 30th June 2019

Accepted 30th July 2019

DOI: $10.1039 / c 9 r a 04927 d$

rsc.li/rsc-advances

\section{Application of ANN modeling techniques in the prediction of the diameter of PCL/gelatin nanofibers in environmental and medical studies}

\begin{abstract}
Saba Kalantary, ${ }^{a}$ Ali Jahani, (D) *b Reza Pourbabaki ${ }^{\mathrm{a}}$ and Zahra Beigzadeh ${ }^{\mathrm{c}}$
Prediction of the diameter of a nanofiber is very difficult, owing to complexity of the interactions of the parameters which have an impact on the diameter and the fact that there is no comprehensive method to predict the diameter of a nanofiber. Therefore, the aim of this study was to compare the multi-layer perceptron (MLP), radial basis function (RBF), and support vector machine (SVM) models to develop mathematical models for the diameter prediction of poly( $\varepsilon$-caprolactone) (PCL)/gelatin (Gt) nanofibers. Four parameters, namely, the weight ratio, applied voltage, injection rate, and distance, were considered as input data. Then, a prediction of the diameter for the nanofiber model (PDNFM) was developed using data mining techniques such as MLP, RBFNN, and SVM. The PDNFM MLP is introduced as the most accurate model to predict the diameter of PCL/Gt nanofibers on the basis of costs and time-saving. According to the results of the sensitivity analysis, the value of the PCL/Gt weight ratio is the most significant input which influences PDNFM MLP in PCL/Gt electrospinning. Therefore, the PDNFM model, using a decision support system (DSS) tool can easily predict the diameter of PCL/Gt nanofibers prior to electrospinning.
\end{abstract}

\section{Introduction}

In recent decades, nanofibers have been proposed as excellent candidates in many areas owing to their unique properties such as their micro or nanoscale structure, high porosity, large surface area to volume ratio, and their substantial mechanical flexibility and strength in the medical and engineering industries, including drug delivery, filtration, tissue engineering, protective clothing, nanoelectronics, nanobiosensors, nanocatalysis, and so forth..$^{1,2}$ Electrospinning is an easy, versatility, and inexpensive method to prepare polymeric ultrafine fibers under the action of a high electrostatic field from a wide variety of polymers. ${ }^{3,4}$ Gelatin (Gt) is one of the most common biopolymers, and has been widely explored owing to its biodegradability, biocompatibility, immunogenicity profiles, hydrophilic nature, and commercial availability at a low $\operatorname{cost} .^{5} \mathrm{Poly}\left(\varepsilon^{-}\right.$ caprolactone) (PCL) is a semi-crystalline linear hydrophobic polymer which is most commonly used for medical and environmental applications, owing to its biocompatibility and slow biodegradability. ${ }^{6}, 7$ Polymer blending is one of the most effective techniques for providing new or desirable properties such

${ }^{a}$ Department of Occupational Health Engineering, School of Public Health, Tehran University of Medical Sciences, Tehran 1416753955, Iran

${ }^{b}$ Department of Natural Environment and Biodiversity, Faculty of Environment, College of Environment, Karaj 31746118, Iran.E-mail: ajahani@alumni.ut.ac.ir

${ }^{c}$ Environmental Health Engineering Research Center, Kerman University of Medical Sciences, Kerman 7616913555, Iran as blending synthetic and natural polymers. Therefore, gelatin can be blended with PCL to improve the biomechanical, degradation, and cell adhesion properties. ${ }^{6}$

Although a wide variety of complicated electrospinning techniques such as coaxial, ${ }^{8}$ modified coaxial, ${ }^{9}$ tri-axial, ${ }^{10}$ side-by-side ${ }^{11}$ and other complicated techniques ${ }^{12}$ have been successively reported, only a few limited publications have reported manipulation of the quality of the nanofibers, which is often evaluated by their diameter. Fiber size distribution and morphology play a significant role in the porosity, the surface to volume ratio, functionality, and performance. ${ }^{13}$ The shape, diameter of the electrospun nanofibers, and spatial distribution have a close relationship with their functional performance and can be divided into four categories: polymer properties (molecular weight and solubility), properties of the working fluids (the concentration, solution viscosity, dielectric properties, surface tension, and conductivity), the operational conditions (applied voltage, the fluid flow rate, nozzle-collector distance, and nozzle diameter of the spinneret), and manipulation of the environmental conditions (temperature, atmospheric pressure, relative humidity, the possible vacuum and even hot air blowing). These parameters have a positive impact on downsizing of the nanofibers. ${ }^{14-18}$ Despite important experimental investigations to determine fiber diameter, using scanning electron microscopy, transmission electron microscopy, and atomic force microscopy for example, it is still time-consuming and expensive. ${ }^{18,19}$ Furthermore, complexities in the electrospinning method and many factors simultaneously affecting the preparation techniques cause the findings from 
statistical tools, such as response surface methodology and regression analysis, to be very noisy. ${ }^{\mathbf{1 6}, 20}$ Regression analysis is one of the traditional techniques that has been used for model generation but the accuracy decreases when the independent parameters increase. In complex phenomena modeling, methods such as an artificial neural network (ANN) are employed. ${ }^{21}$ An ANN is an attractive and flexible choice for solving linear and nonlinear multivariate regression and different problems because it is based on the natural neural network of the brain..$^{21-23}$ An ANN consists of interconnected processing elements, such as an input layer, various hidden layers and an output layer which is capable of learning from samples, using transfer functions between neurons and a specific learning algorithm in the structure of a program without being affected by data noise. ${ }^{24-26}$ Nowadays, different models and learning algorithms can be applied to modeling and controlling the electrospinning processes. ${ }^{27,28}$ In this paper, we have compared the multi-layer perceptron (MLP), radial basis function (RBF), and support vector machine (SVM) models for predicting the diameter of PCL/gelatin nanofibers. The main objectives were to: (i) analyze the effects of weight ratios, applied voltage, injection rate, nozzle-collector distance, and their individual and interactive effects on the diameter of PCL/gelatin nanofibers; (ii) compare different data mining models to identify the most accurate model; (iii) detect the most significant factors affecting the diameter of PCL/gelatin nanofibers using sensitivity analysis; and (iv) design a decision support system (DSS) for predicting the diameter of electrospun PCL/Gt nanofibers.

\section{Experimental}

\subsection{Materials}

Poly( $\varepsilon$-caprolactone) $\left(M_{\mathrm{w}}=80000 \mathrm{~g} \mathrm{~mol}^{-1}\right)$, gelatin from porcine skin type A (gel strength $300 \mathrm{~g}$ bloom), glacial acetic acid (AA), and formic acid (FA) were all provided by Sigma-Aldrich.

\subsection{Preparation of the polymer solution}

A separate solution was prepared from PCL and gelatin by dissolving $15 \% \mathrm{w} / \mathrm{w}$ of the sample in AA : FA in a $9: 1$ ratio using a magnetic stirrer at room temperature for $4 \mathrm{~h}$. Following this, PCL and gelatin (PCL/Gt) were mixed at seven different volume ratios $(80: 20,70: 30,60: 40,50: 50,40: 60,30: 70$ and $20: 80$ ) for $20 \mathrm{~h}$ prior to electrospinning. ${ }^{29}$

\subsection{Electrospinning}

The prepared PCL/Gt solutions were added to a plastic syringe with a needle tip of size $23 \mathrm{G}$. The injection rate of the polymer solution was $0.6-2 \mathrm{ml} \mathrm{h}^{-1}$, and the distance between the needle tip and collector was $5-20 \mathrm{~cm}$. The high voltage was $6-22 \mathrm{kV}$. $^{30}$

\subsection{Characterization}

Scanning electron microscopy (SEM, DSM-960A Model, ZEISS, Germany) was applied to investigate the morphology of the electrospinning nanofibers at an accelerating voltage of $20 \mathrm{kV}$. Before SEM, the samples were coated with gold. For each sample, the average fiber diameter was determined from about 70 random measurements using Image J software.

\subsection{Data collection}

Different parameters affecting the diameter of electrospinning nanofibers of PCL/Gt were measured, five independent input electrospinning parameters, namely: $\mathrm{PCL} / \mathrm{Gt}$ weight ratios $\left(X_{1}, \mathrm{wt} \%\right)$; electrospinning voltage $\left(X_{2}, \mathrm{kV}\right)$; needle-to-collector distance $\left(X_{3}\right.$, $\mathrm{cm})$; injection rate of the solution $\left(X_{4}, \mathrm{ml} \mathrm{h}^{-1}\right)$; and the output data include average diameter of PCL/Gt nanofiber $(Y, \mathrm{~nm})$ were chosen and their effects on the diameter size of electrospinning PCL/Gt were investigated using an artificial intelligence procedure.

\subsection{MLP neural network}

The MLP model is a multi-layer network technique for modeling and prediction. The MLP uses a self-learning mechanism using samples for modeling nonlinear and complex process of the real world. Thus, it has been applied as the benchmark model by many researchers. ${ }^{31-33}$ The MLP network architecture contains an input layer, hidden layers, and an output layer for an accurate prediction of the fiber diameter. An MLP uses some computational processing elements (PEs) which are called neurons. The learning algorithm is a process of updating the connection weights of the neurons continuously in hidden layers to minimize the differences between the target and network output samples. ${ }^{34}$

In this study, three activation functions consisting of a hyperbolic tangent, logarithmic sigmoid, and linear transfer functions were examined to optimize the prediction of the diameter of the electrospinning PCL/Gt nanofibers model.

The backpropagation (BP) method is found to be the most popular and powerful nonlinear statistical method, therefore it is an effective technique for calculating the weight and biases of neurons. The BP algorithm uses learning rules to assign weight arrangements of neurons and layers to nodes based on the output of the network. The weights alter during the learning, and the process is repeated until the best performance is achieved, and the learning process will end. ${ }^{21,35}$

The aim of BP is to minimize the error between $Y$ (average diameter of PCL/Gt nanofiber) and $Y_{\text {net }}$ (MLP output) in which $X$ and $Y$ are given to the network and the weight of the PEs $(w)$ and input samples $(X)$ are adjusted, and an output of the $j$ th PE on the $k$ th $\left(\mathrm{PE}_{j}^{k}\right)$ is calculated using eqn (1):

$$
\text { net }_{j}{ }^{k}=\sum_{i=0}^{n} w_{j i} x_{j i}
$$

The specific functions known as the transfer or threshold functions are introduced to the network, and the output value of the neurons is presented in eqn (2):

$$
Y_{\text {net }}=\int \text { net }_{j}
$$

In the next step, the weights of the $t$ numbers of the input/ output parts ( $X$ and $Y$ ) will be changed using the delta rule in eqn (3):

$$
w_{j i}^{t}=w_{j i}^{t-1}+\Delta w_{j i}^{t}
$$


Several of the learning algorithms for BP have been used to end the learning process and for adjusting weights. In this paper, the validation data set performed was the generalization of MLP and avoids overtraining of the network. The final step is the test performance of the MLP using the test data set, which is not used in the training and validation data sets. To perform this, the samples were randomly divided into three subsets which include the training data set with $60 \%$ of the total samples, a validation data set with $20 \%$ of the total samples and a test data set with $20 \%$ of the total samples.

\subsection{Radial basis function neural network}

The radial basis function neural networks (RBFNNs) have a fast learning algorithm and simple topological structure, they have been structured with an input, hidden, and output layer, such as in the MPL, but in comparison they are different in the matter of the activation function in the hidden layer. The RBFNNs have a fast learning algorithm, high accuracy, and a unifying link in the function approximation amongst many different research fields in recent years. ${ }^{1,36,37}$ The radial basis function neural network (RBFNN) has two types of data sets, which are training and testing. The application of radial function in the neurons of the hidden layer, the number of RBFNNs, the corresponding centers, and the output layer weight matrix is different in RBFNNs. ${ }^{36,38}$ The application of the first layer in a RBFNN network is the distribution of the variables to the hidden layer. Each neuron of the hidden layer creates a radial classifier and spheres of answers in the multidimensional decision space, and the number of neurons depends on the problem to be solved. ${ }^{36}$ The most common basis function, in RBFNN structure, is a Gaussian function that is characterized by a center and peak width ${ }^{39}$ as given by eqn (4):

$$
R_{j}(x)=\exp \left(\frac{\left\|x-a_{j}\right\|^{2}}{2 \sigma^{2}}\right)
$$

In which, $R_{j}(x)$ is the radial basis function, $\left\|x-a_{j}\right\|$ which represents the determined Euclidean distance between the total of $a_{j}$ (RBF function center), $x$ known as (input vector or variables), and $\sigma$ is a positive real number, respectively..$^{36}$ Finally, the output layer should be calculated using eqn (5):

$$
y_{k}=\sum_{j=1}^{m} w_{j k} R_{j}(x)+b_{j}
$$

In which $w_{j k}$ is the weights of the neurons, $j$ is the number of each node in the hidden layer, $m$ is the number of neurons, and $b_{j}$ represents bias. The value of $w_{j k}$ is adjusted to reduce the mean square error of the output when the training of the network ends. At the end of the training and after fixing the number of neurons and the weights, the performance of the network will be defined. ${ }^{36,40}$

\subsection{Support vector machine}

The SVM, as a standard tool for machine learning and data mining, was introduced for solving a variety of learning, classification, and prediction problems. ${ }^{41,42}$ The SVM seeks the widest possible margin between the boundaries of classes to minimize the uncertainty of classification. This method of prediction minimizes the probability of over-fitting in decisions, which limits the prediction of the diameter of nanofibers. ${ }^{\mathbf{4 3 , 4 4}}$

There are two data sets, including training and testing in the structure of SVM. The values of the target are based on an $n$ dimensional matrix in which the most accurate boundaries and margins possible are available. ${ }^{\mathbf{4 3 4}}$

The SVM model algorithm equation can be expressed in eqn (6):

$$
y(x)=\sum_{i=1}^{n} \alpha_{i} K\left(x_{i}, x_{j}\right)+b
$$

In which $y(x)$ is the SVM output, $\alpha_{i}$ is a multiplier, $K$ presents the kernel function, and $b$ is the threshold parameter.

Next, we provide the Gaussian RBF in eqn (7), as the activation function in this study. As is known, RBF is the most common function with a considerable ability to control the generalization of the SVM network.

$$
K\left(x_{i}, x_{j}\right)=\exp \left(-\gamma\left\|x_{i}-x_{j}\right\|^{2}\right)
$$

In which $x_{i}$ and $x_{j}$ are samples and $\gamma$ is the kernel parameter, respectively.

To achieve the most accurate SVM for predicting the fiber diameter of electrospinning PCL/Gt, eqn (8) should be minimized.

$$
\frac{1}{2}\|w\|^{2}+C \sum_{i=1}^{n} \xi_{i}
$$

In which $\frac{1}{2}\|w\|^{2}$ presents the margin, $\sum \xi_{i}$ is the training errors, and $C$ is the tuning parameter respectively.

\subsection{Model selection}

To evaluate the performance of the model that is not applied in the training process, a simulation of the test data set was used. The performance of the designed MLP, RBFNN, and SVM was assessed using several statistical indicators such as the mean squared error (MSE, eqn (9)), the root mean squared error (RMSE, eqn (10)), the mean absolute error (MAE, eqn (11)), the coefficient of determination $\left(R^{2}\right.$, eqn (12)), Willmott's index (WI, eqn (13)), and the Taylor diagram. ${ }^{45-48}$

$$
\begin{aligned}
\mathrm{MSE} & =\frac{\sum_{i=1}^{n}\left(y_{i}-\hat{y}_{i}\right)^{2}}{n} \\
\mathrm{RMSE} & =\sqrt{\frac{\sum_{i=1}^{n}\left(y_{i}-\hat{y}_{i}\right)^{2}}{n}}
\end{aligned}
$$

$$
\text { MAE }=\frac{\sum_{i=1}^{n}\left|y_{i}-\hat{y}_{i}\right|}{n}
$$



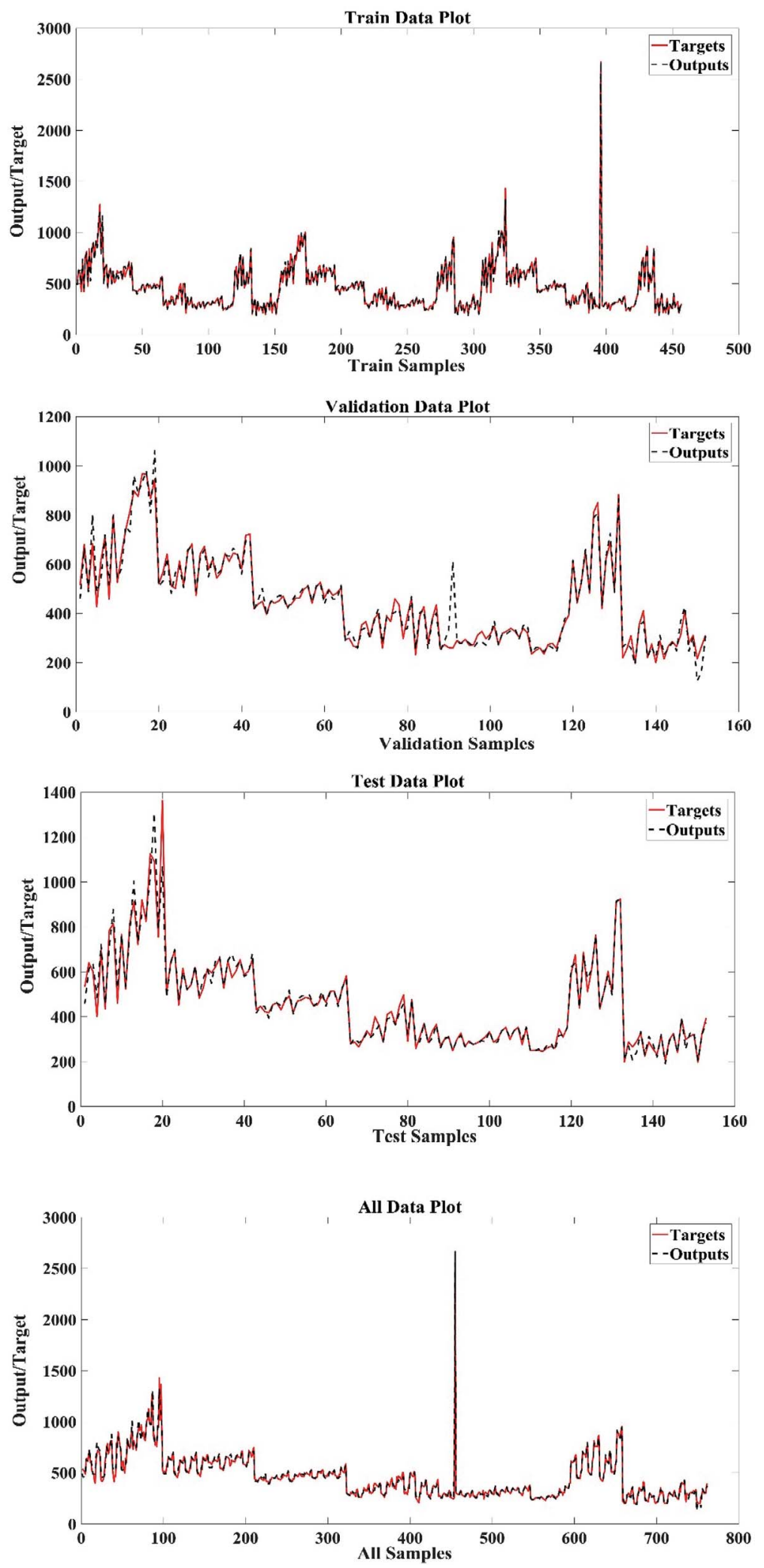

Fig. 2 Target and output nanofiber diameter values for training, validation, and testing sets and all data.

distance), as input variables, and the effects of these parameters on the diameter of the PCL/gelatin nanofibers as outputs, were entered into the software MATLAB R2016b to design the most accurate prediction of the diameter for the nanofiber model
$(\mathrm{PDNFM})_{\mathrm{MLP}}$. In the optimization of the MLP model parameters, the total number of samples (762 samples) was randomly divided into three data sets. The training data subset contained $60 \%$ of all samples (457 samples), the validation data set 

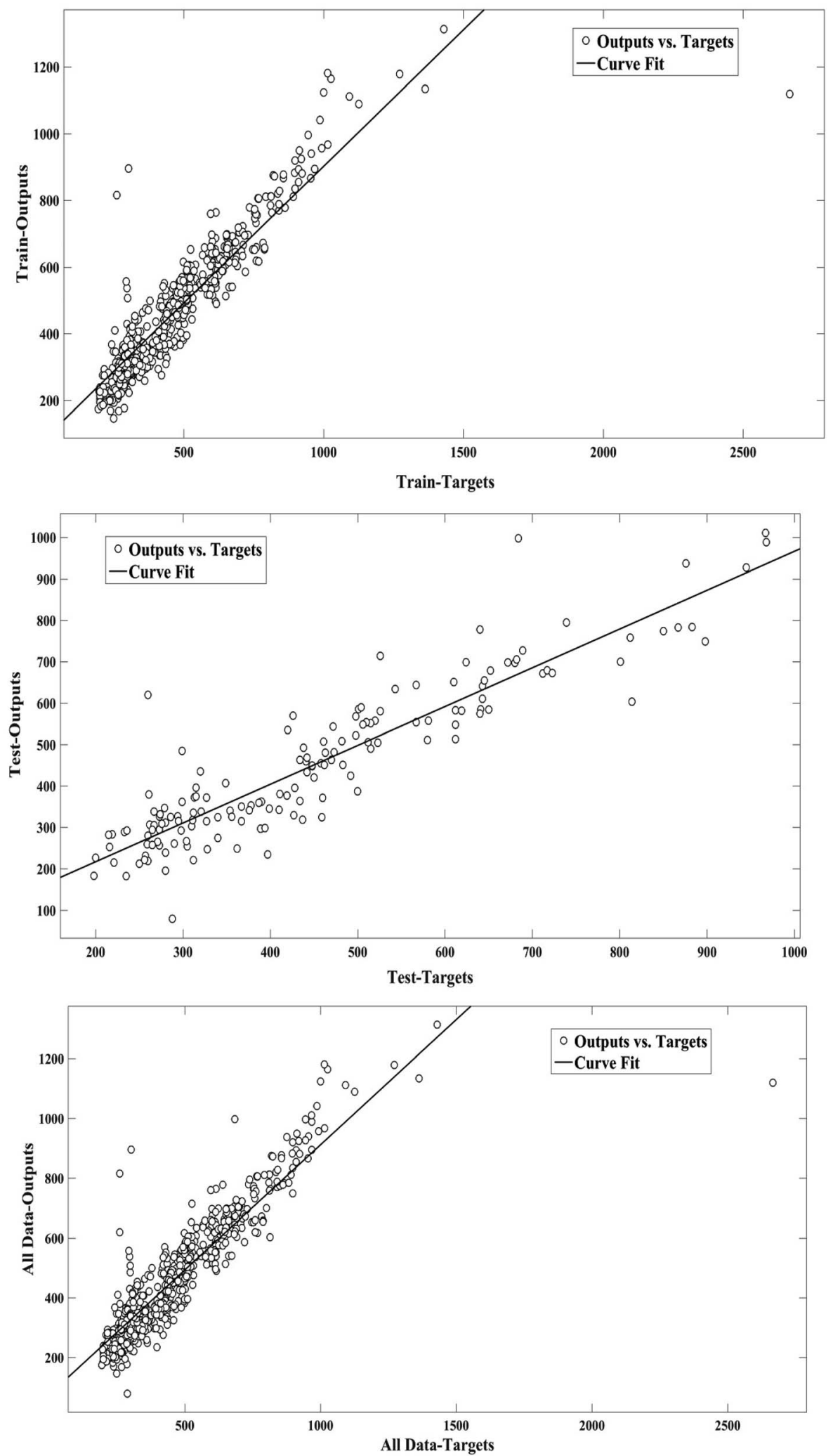

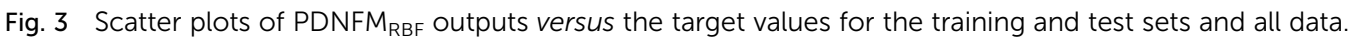

space that separates all data in this multi-dimensional space more easily. ${ }^{49,50}$

The SVR regression performance is associated with the proper selection of the parameters, which are $\varepsilon, C$, and $\gamma$. The value of the $\varepsilon$ is related directly to the number of support vectors. ${ }^{51}$ The value of $\gamma$ is determined by the width of the bellshaped curves in the structure of the SVM regression with a Gaussian function (as shown in eqn (7)). In this research, the 

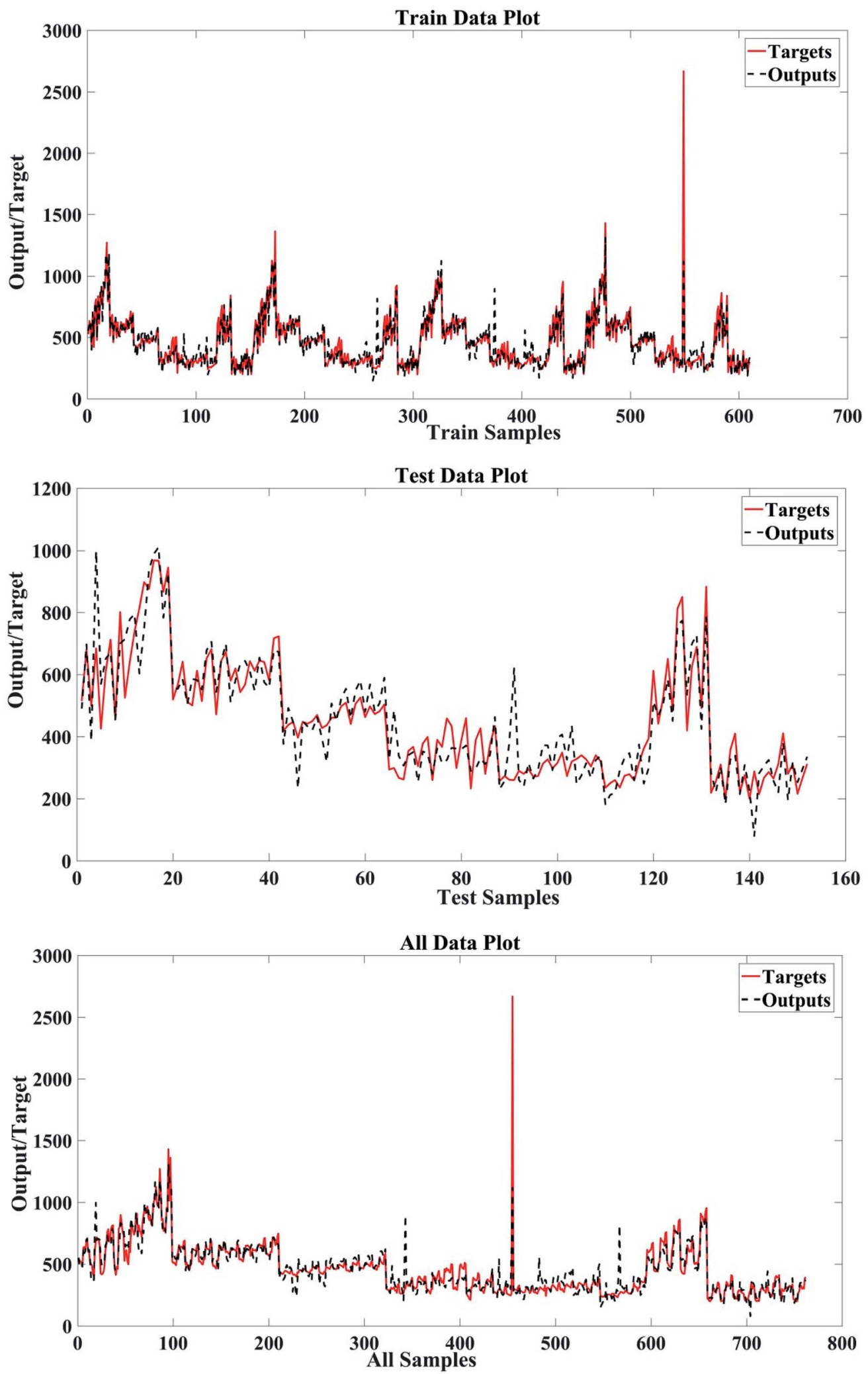

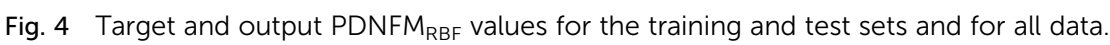

polynomial kernel function was found to be more accurate than the Gaussian function; therefore, we did not achieve the value of $\gamma$. On the other hand, the value of $C$ allows the SVM to gain more simple curves as the goal accuracy is obtained. ${ }^{42}$ Thus, we selected the values of the $\varepsilon$ and $C$ parameters to achieve a highly accurate SVM regression in the prediction of the diameter of the 

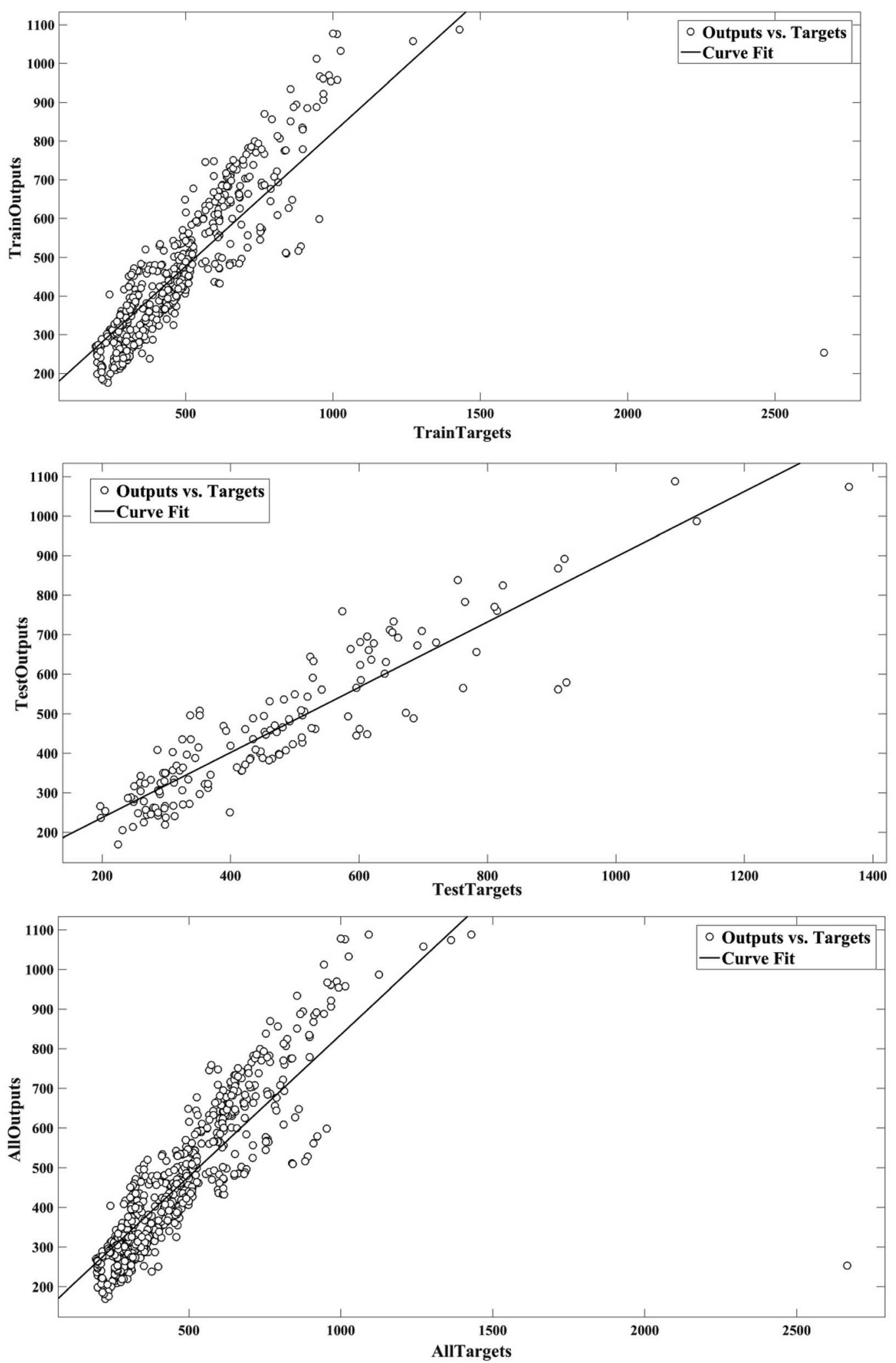

Fig. 5 Scatter plots of PDNFM SVM $_{\text {Onts }}$ outs versus the target values for the training and test sets and for all data.

nanofibers. Thus, a sensitivity analysis of the predicted outputs of the optimal PDNFM $_{\mathrm{MLP}}$ was conducted. In the sensitivity analysis, each parameter in the range of the standard deviation with 50 steps were changed, while the other parameters were fixed at the value of the average. Next, the standard deviation of the outputs for each parameter change were measured as the PDNFM $_{\text {MLP }}$ sensitivity for that parameter. As illustrated in Fig. 9, the PDNFM $\mathrm{MLP}_{\mathrm{M}}$ sensitivities for the input variables have 

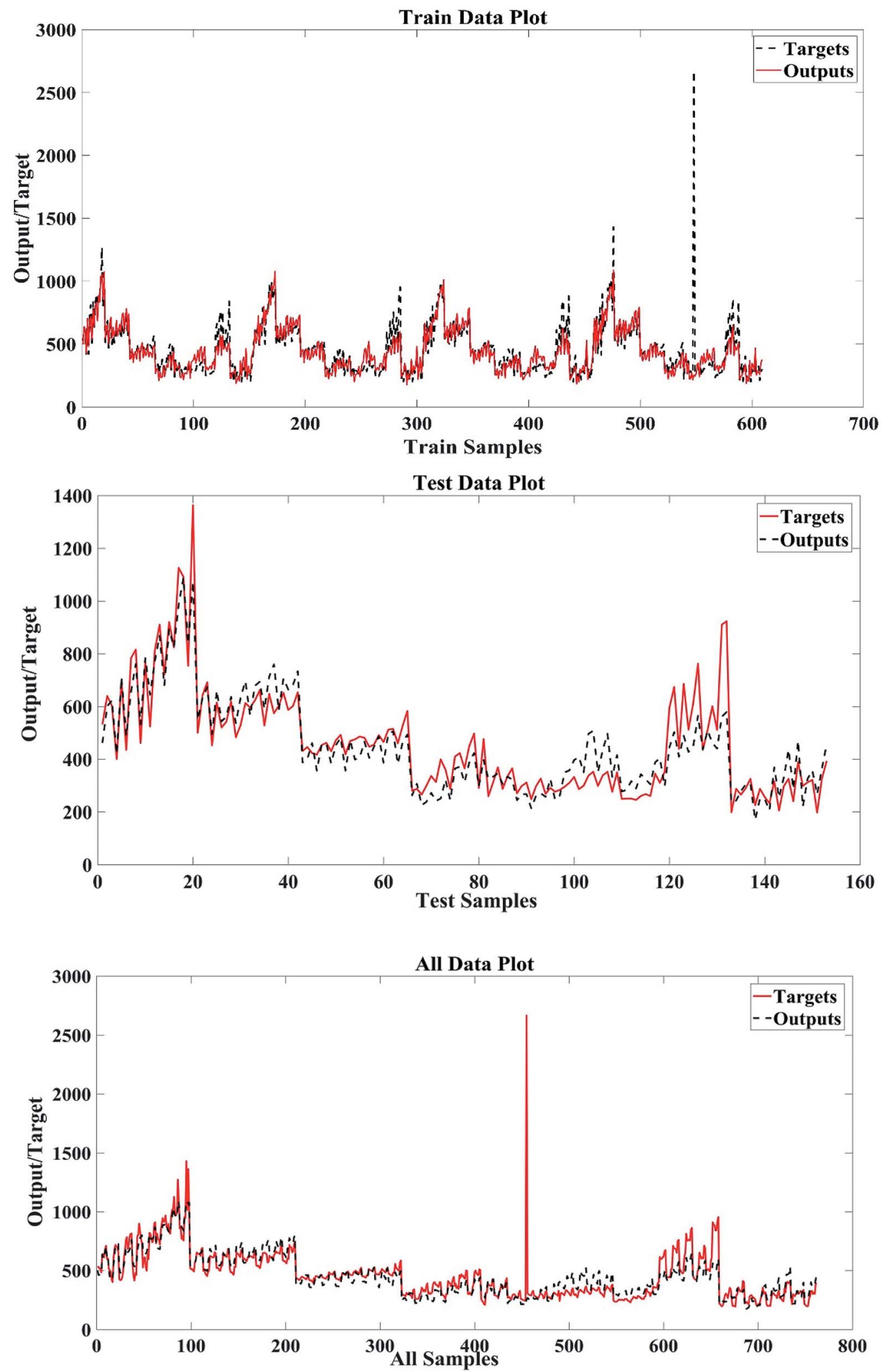

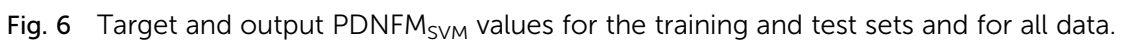

been explored using sensitivity analysis. The results of the sensitivity analysis demonstrate that the electrospinning processing parameters play a primary role in the MLP outputs. As can be seen from Fig. 9, the value of the PCL/Gt weight ratios is detected as the most significant input influencing the PDNFM $_{\text {MLP }}$ output. 


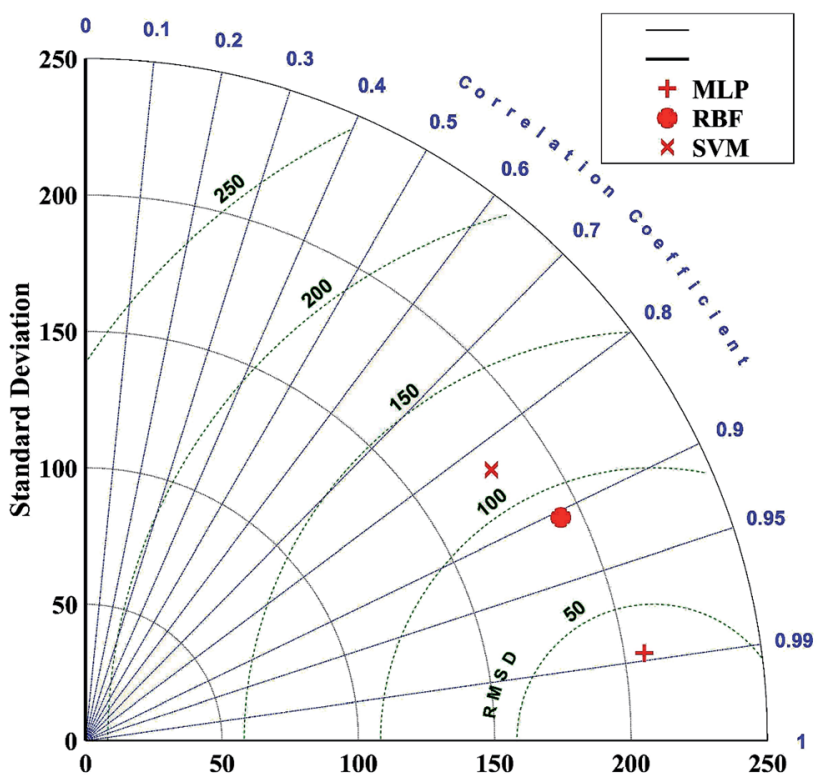

Fig. 7 Taylor diagrams for evaluating the performances of the computing models (MLP, RBFF, SVM).

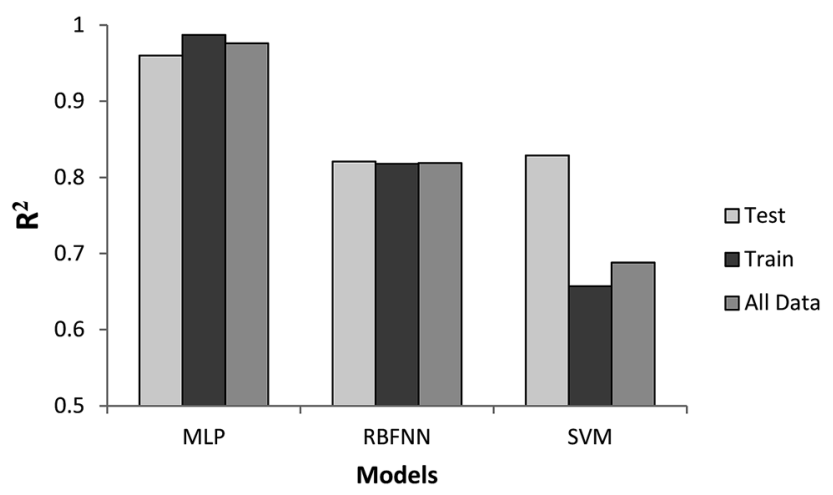

Fig. 8 The performance measures of the designed PDNFMs.

Fig. 10a and c shows the effect of the PCL/Gt weight ratios and applied voltage on the diameter of the fiber. As one can see, there is a negative correlation between the weight ratio and the

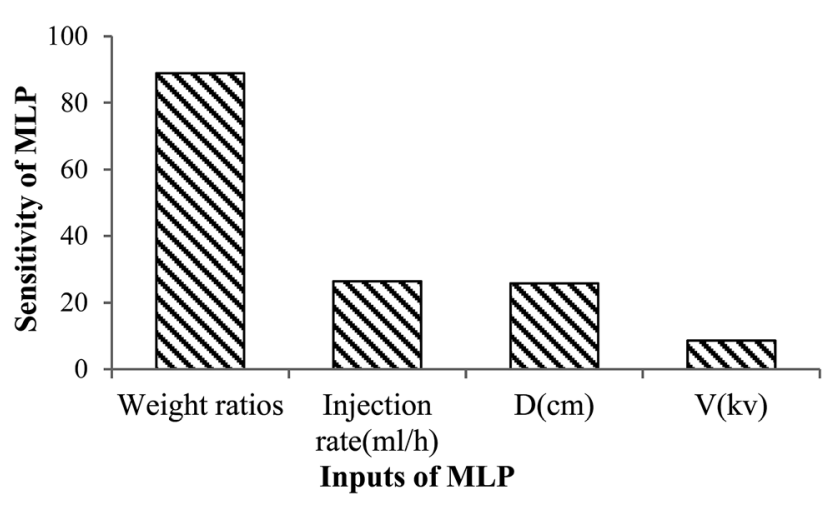

Fig. 9 Sensitivity analysis of PDNFM voltage and fiber diameter, therefore the fiber diameter decreases with the increased voltage or weight ratios. The fiber diameter decreased upon increasing the content of PCL in the AA/FA solution, as shown in Fig. 10a. A reason for this result could be the presence of an emulsion, which can be weakened at higher PCL contents. PCL/Gt exhibit an emulsion structure when using an AA or AA/FA mixture as a solvent. The explanation for this emulsion structure is the absence, or very limited miscibility, of Gt and PCL and the relatively weak interaction with AA and FA. These results are in accordance with those obtained by Denis et al. ${ }^{29}$ Also, a decrease in the viscosity of the polymer solution can be ascribed as the cause of the decrease in the nanofiber diameter. ${ }^{57}$ As shown in Fig. 10b, the effect of distance on the diameter of the nanofiber is contradictory, the fiber diameter increases, as well as decreases, with an increase of the distance. Indeed, at a short spinning distance, there will not be sufficient time for the solvent to evaporate before the jet is placed on the collector owing to thicker nanofibers. Furthermore, the diameter of the nanofibers decreased with an increase in the spinning distance. ${ }^{58}$ The curve is downwardsloping for high values of the spinning distance. This result may be explained by the fact that by increasing the spinning distance, the jet has enough time to stretch and the solvent will have more time to evaporate before the jet is deposited on the collector leading to thinner fiber formation. ${ }^{58}$ Another reason is probably owing to breaking of the formed jet into two or more jets, leading to finer nanofibers. ${ }^{20}$ These results were reported in recent studies. ${ }^{56-58}$ Fig. 10c shows the effect of applied voltage on the diameter of the nanofibers. In fact, at a high applied voltage, the electric field strength is high, resulting in further stretching of the jet before it is deposited on the collector, and hence the fiber diameter will decrease. On the other hand, increasing the applied voltage will result in an increased surface charge on the droplet jet, favoring the formation of thinner fibers. This observation is in agreement with those from previously published reports. ${ }^{59-61}$

Considering the trends observed in Fig. 10d, the injection rate has a two-fold impact on the diameter of the fiber. First, the diameter of the nanofibers increases with an increase in the volume injection rate. The published literature indicates that an increase in the injection rate of the solution typically increases the diameter of the nanofibers. ${ }^{\mathbf{6 2}, 63}$ This result could be explained by the fact that an increase in injection rate leads to an increase of the amount of polymer solution delivered to the top of the needle making the jet diameter increase. ${ }^{57,58,62}$ Therefore there is a limitation to this, after reaching an optimum value, the diameter of the nanofibers continuously decrease with an increase in the injection rate. Some studies show that the injection rate increase leads to a decrease in the diameter of the nanofibers. ${ }^{20,64}$ As increasing the injection rate will cause higher electrostatic forces, an increase in the volumetric charge density on the droplet jet, and a greater tensile force which might increase the stretching of the jet, resulting in the formation of thinner diameter nanofibers. ${ }^{59,65}$ The literature shows that ANN techniques have been designed with data analysis, modeling, simulation, and output. ${ }^{21}$ These models have been used to investigate the relationship between electrospinning parameter and the diameter of the nanofibers or fiber morphology.$^{1,58}$ 

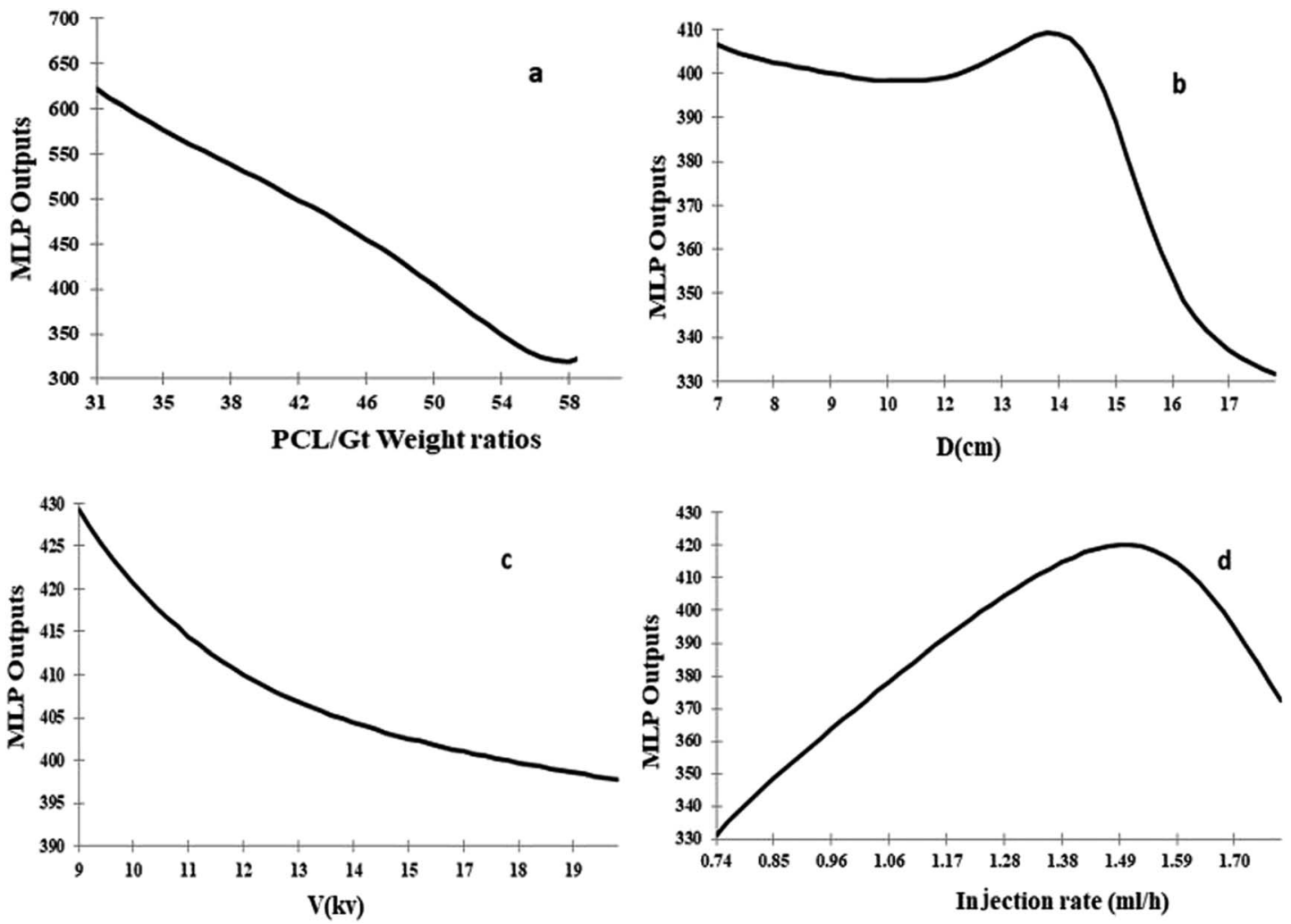

Fig. 10 The trend of the DPNFM MLP output changes upon varying: the (a) PCL/Gt weight ratio; (b) distance; (c) voltage; and (d) injection rate.

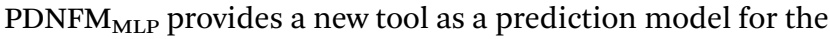
electrospinning of nanofiber considering the variables of the PCL/Gt weight ratios, applied voltage, distance and, injection

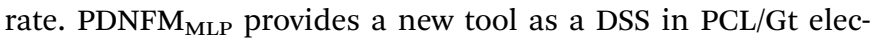
trospinning for prediction of the diameter of the nanofibers resulting from electrospinning. PDNFM $_{\mathrm{MLP}}$ was developed for researchers or lab technician to predict the diameter of PCL/Gt nanofibers, which helps them to save time and money. In this study, which develops a tool for the prediction of the diameter of PCL/Gt nanofibers, the steps of model development and implementation are described as a DSS. We proposed the flow diagram shown in Fig. 11 to design a DSS using PDNFM $_{M L P}$ for PCL/Gt electrospinning. The described DSS has been designed using data analysis, modeling, simulation, and output. The

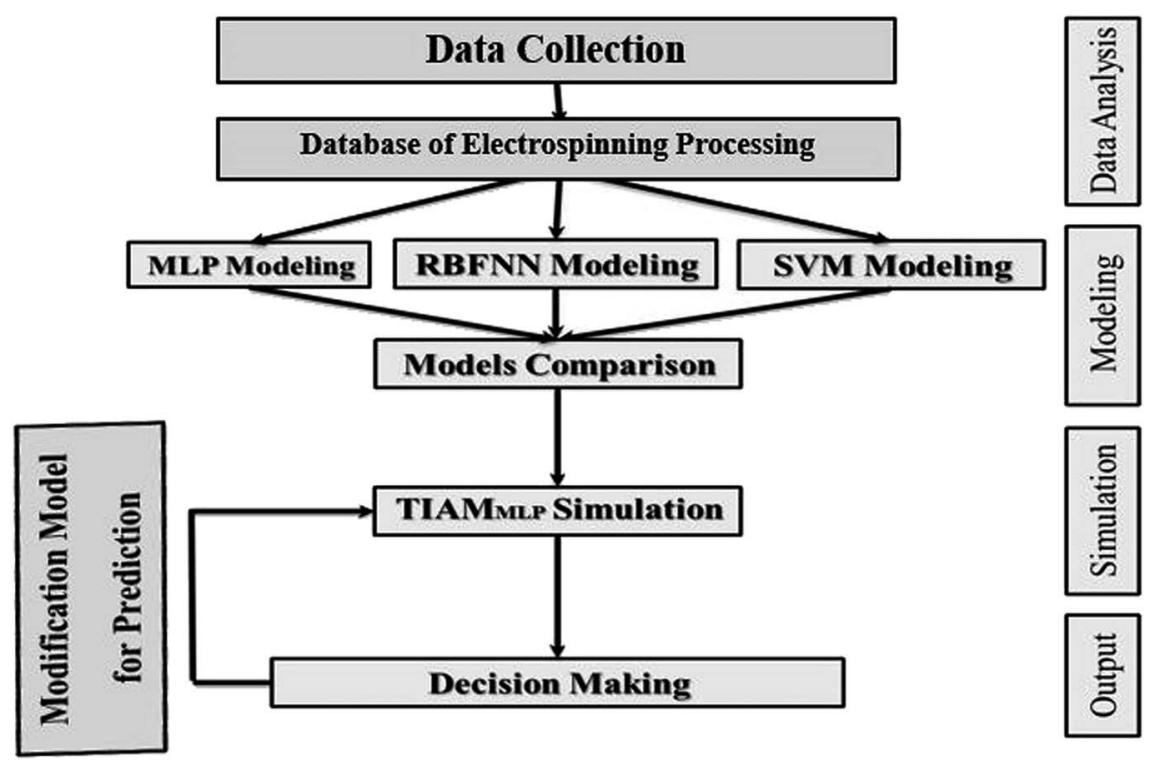

Fig. 11 Flow diagram for the development of a DSS for PCL/Gt electrospinning. 

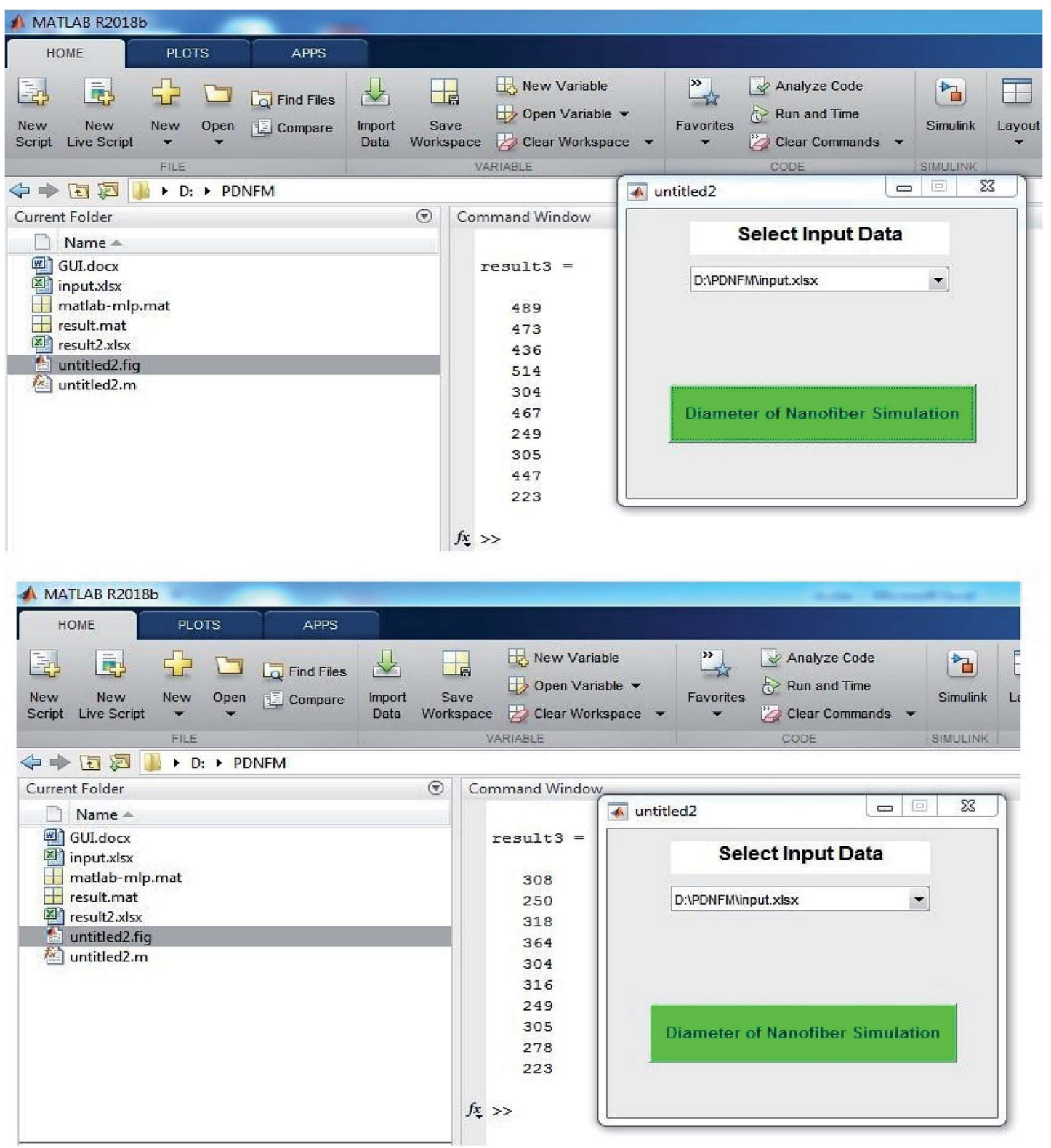

Fig. 12 Two different electrospinning processing parameters for the diameter of PCL/Gt nanofibers.

output of PDNFM $\mathrm{MLP}$ is applicable in the electrospinning of $\mathrm{PCL} / \mathrm{Gt}$ nanofibers, and the electrospinning processing parameters and diameter of PCL/Gt nanofibers could be modified based on the proposed DSS to reduce time and costs. The values of the optimization factors are not proposed for use in other research, but they could be used as alternatives values to optimize models in this kind of research. Finally, a graphical user interface (GUI) was designed to run the PDNFM model on new data for which the researchers are planning for PCL/Gt electrospinning. A GUI, as a user friendly tool, is designed to provide easy utilization of an ANN technique. The GUI provides a tool to predict nanofiber diameter before electrospinning by changing the values of the input parameters, which saves time and material. ${ }^{66}$
In general, it is necessary to mention that sizes at the nanoscale significantly impact the structural, mechanical, thermal, thermo-dynamic, kinetic, and electrical properties of materials. ${ }^{28}$ Furthermore, rapidly increasing interest has been shown by users, and this has led to the production of diverse nanofibers for versatile usage in various applications. ${ }^{67}$ Determination of the nanofiber size is, therefore, a matter of considerable importance in electrospinning for various applications. ${ }^{68}$ With the help of the PDNFM model, the changes are detectable before electrospinning. This means that a change in the diameter of the nanofibers and the morphology is measurable before electrospinning. The researchers, engineers, and experts working in academia and industry can easily predict the diameter of nanofibers in electrospinning. GUI as a DSS tool will be run on new data by simply inputting the diameter of the 
nanofiber, as shown in Fig. 12. As an example, Fig. 12 illustrates the effect of two different electrospinning processing parameters on the diameter of PCL/Gt nanofibers. We found the diameter of nanofibers to be thick (200 to $500 \mathrm{~nm}$ ) in plan (a) for our specific application in PCL/Gt electrospinning. Therefore, we modified the electrospinning processing parameters of plan (a) to plan (b), in which the diameter of the nanofibers will be optimal in PCL/Gt electrospinning for our application. The modification was conducted by changing the most significant factors in the sensitivity analysis results and trends shown in Fig. 10. Overall, the findings of this study indicate the advantages of modeling and optimizing the diameter of electrospun nanofibers using an ANN and the advantages of these models for experts in the nano-field, tissue engineering, pharmaceutical, environmental, medical, food and engineering industries to help reduce product costs.

\section{Conclusions}

In this research, MLP, RBFNN, and SVM models were developed to predict the diameter of a PCL/Gt nanofiber considering variables such as the PCL/Gt weight ratios, applied voltage, distance, and injection rate. The performance of the designed MLP, RBFNN, and SVM models was evaluated by root mean squared error, mean absolute error, coefficient of determination, Willmott's index, and a Taylor diagram. Furthermore, the sensitivity for each electrospinning parameter has been explored using sensitivity analysis. In the structure provided using MATLAB software, the results indicated the greater accuracy of the MLP compared with the RBFF and SVM models and showed the potential of the MLP modeling technique as a handy tool for predicting the ideal electrospinning process parameters and nanofiber properties. It also showed that the PCL/Gt weight ratio is the most significant input which influences the MLP output. It is recommended to apply a modeling approach such as the MLP model to estimate and optimize the size of nanofibers before electrospinning. This can be worthwhile in terms of the economy, time, and scientific aims. It can thus be suggested that designing and applying the prediction method for different kind of nanofibers or nanomaterials should be performed using ANN techniques for various applications in environmental, medical and other industries.

\section{Conflicts of interest}

There are no conflicts to declare.

\section{Acknowledgements}

The authors would like to acknowledge the Tehran University of Medical Sciences and the College of Environment.

\section{Notes and references}

1 K. Nasouri, Novel estimation of morphological behavior of electrospun nanofibers with artificial intelligence system (AIS), Polym. Test., 2018, 69, 499-507.
2 M. Naghibzadeh and M. Adabi, Evaluation of effective electrospinning parameters controlling gelatin nanofibers diameter via modelling artificial neural networks, Fibers Polym., 2014, 15(4), 767-777.

3 B. Feng, S. Wang, D. Hu, W. Fu, J. Wu, H. Hong, et al., Bioresorbable electrospun gelatin/poly(e-caprolactone) nanofibrous membrane as a barrier to prevent cardiac postoperative adhesion, Acta Biomater., 2019, 83, 211-220.

4 W. P. Lu and Y. Guo, Electrospinning of Collagen and Its Derivatives for Biomedical Applications, Novel Aspects of Nanofibers, IntechOpen, 2018.

5 M. E. Hoque, T. Nuge, T. Yeow and N. Nordin, Electrospinning of gelatin nanofibre: current trends in tissue engineering applications, J. Appl. Mech. Eng., 2013, 2, e122.

6 L. Ghasemi-Mobarakeh, M. P. Prabhakaran, M. Morshed, M.-H. Nasr-Esfahani and S. Ramakrishna, Electrospun poly( $\varepsilon$-caprolactone)/gelatin nanofibrous scaffolds for nerve tissue engineering, Biomaterials, 2008, 29(34), 4532-4539.

7 L. Van der Schueren, B. De Schoenmaker, Ö. I. Kalaoglu and K. De Clerck, An alternative solvent system for the steady state electrospinning of polycaprolactone, Eur. Polym. J., 2011, 47(6), 1256-1263.

8 Y. H. Wu, D. G. Yu, H. P. Li, X. Y. Wu and X. Y. Li, Medicated structural PVP/PEG composites fabricated using coaxial electrospinning, e-Polym., 2017, 17(1), 39-44.

9 Q. Wang, D. G. Yu, L. L. Zhang, X. K. Liu, Y. C. Deng and M. Zhao, Electrospun hypromellose-based hydrophilic composites for rapid dissolution of poorly water-soluble drug, Carbohydr. Polym., 2017, 174, 617-625.

10 C. Yang, D. G. Yu, D. Pan, X. K. Liu, X. Wang, S. A. Bligh and G. R. Williams, Electrospun pH-sensitive core-shell polymer nanocomposites fabricated using a tri-axial process, Acta Biomater., 2016, 35, 77-86.

11 K. Wang, X. K. Liu, X. H. Chen, D. G. Yu, Y. Y. Yang and P. Liu, Electrospun hydrophilic Janus nanocomposites for the rapid onset of therapeutic action of helicid, ACS Appl. Mater. Interfaces, 2018, 10(3), 2859-2867.

12 D. G. Yu, J. J. Li, M. Zhang and G. R. Williams, High-quality Janus nanofibers prepared using three-fluid electrospinning, Chem. Commun., 2017, 53(33), 4542-4545.

13 M. Naghibzadeh, M. Adabi, H. R. Rahmani, M. Mirali and M. Adabi, Evaluation of the effective forcespinning parameters controlling polyvinyl alcohol nanofibers diameter using artificial neural network, Adv. Polym. Technol., 2018, 37(6), 1608-1617.

14 W. Huang, Y. Hou, X. Lu, Z. Gong, Y. Yang, X. J. Lu, X. L. Liu and D. G. Yu, The Process-Property-Performance Relationship of Medicated Nanoparticles Prepared by Modified Coaxial Electrospraying, Pharmaceutics, 2019, 11(5), 226.

15 X. Wang, L. Zhao, J. Y. Fuh and H. P. Lee, Effect of Porosity on Mechanical Properties of 3D Printed Polymers: Experiments and Micromechanical Modeling Based on XRay Computed Tomography Analysis, Polymers, 2019, 11(7), 1154. 
16 H. Zhou, Z. Shi, X. Wan, H. Fang, D. G. Yu, X. Chen and P. Liu, The Relationships between Process Parameters and Polymeric Nanofibers Fabricated Using a Modified Coaxial Electrospinning, Nanomaterials, 2019, 9(6), 843.

17 B. H. Moghadam, M. Hasanzadeh and A. Haghi, On the contact angle of electrospun polyacrylonitrile nanofiber mat, Bulg. Chem. Commun., 2013, 45, 169-177.

18 M. D. Chomachayi, A. Solouk and H. Mirzadeh, Electrospun silk-based nanofibrous scaffolds: fiber diameter and oxygen transfer, Prog. Biomater., 2016, 5(1), 71-80.

19 S. Rafiei, S. Maghsoodloo, B. Noroozi, V. Mottaghitalab and A. Haghi, Mathematical modeling in electrospinning process of nanofibers: a detailed review, Cellul. Chem. Technol., 2013, 47, 323-338.

20 R. Faridi-Majidi, H. Ziyadi, N. Naderi and A. Amani, Use of artificial neural networks to determine parameters controlling the nanofibers diameter in electrospinning of nylon-6,6, J. Appl. Polym. Sci., 2012, 124(2), 1589-1597.

21 A. Jahani, Forest landscape aesthetic quality model (FLAQM): a comparative study on landscape modelling using regression analysis and artificial neural networks, $J$. For. Sci., 2019, 65(2), 61-69.

22 S. Akbarifard and F. Radmanesh, Predicting sea wave height using Symbiotic Organisms Search (SOS) algorithm, Ocean Eng., 2018, 167, 348-356.

23 H. Aghajani, M. M. Mohadjer, A. Jahani, M. R. Asef, A. Shirvany and M. Azaryan, Investigation of affective habitat factors affecting on abundance of wood macrofungi and sensitivity analysis using the artificial neural network (case study: Kheyrud forest, Noshahr), Iranian Journal of Forest and Poplar Research, 2014, 21(4), 617-627.

24 E. Vatankhah, D. Semnani, M. P. Prabhakaran, M. Tadayon, S. Razavi and S. Ramakrishna, Artificial neural network for modeling the elastic modulus of electrospun polycaprolactone/gelatin scaffolds, Acta Biomater., 2014, 10(2), 709-721.

25 K. Qaderi, S. Akbarifard, M. R. Madadi and B. Bakhtiari, Optimal operation of multi-reservoirs by water cycle algorithm, Proceedings of the Institution of Civil Engineers Water Management, 2017, 171(4), 179-190.

26 A. Jahani, J. Feghhi, M. F. Makhdoum and M. Omid, Optimized forest degradation model (OFDM): an environmental decision support system for environmental impact assessment using an artificial neural network, $J$. Environ. Plan. Manag., 2016, 59(2), 222-244.

27 A. Rabbi, K. Nasouri, H. Bahrambeygi, A. M. Shoushtari and M. R. Babaei, RSM and ANN approaches for modeling and optimizing of electrospun polyurethane nanofibers morphology, Fibers Polym., 2012, 13(8), 1007-1014.

28 A. Jahani, Aesthetic quality evaluation modeling of forest landscape using artificial neural network, Wood \& Forest Science and Technology, 2017, 24(3), 17-22.

29 P. Denis, J. Dulnik and P. Sajkiewicz, Electrospinning and structure of bicomponent polycaprolactone/gelatin nanofibers obtained using alternative solvent system, Int. J. Polym. Mater. Polym. Biomater., 2015, 64(7), 354-364.
30 J. Dulnik, P. Denis, P. Sajkiewicz, D. Kołbuk and E. Choińska, Biodegradation of bicomponent PCL/gelatin and PCL/collagen nanofibers electrospun from alternative solvent system, Polym. Degrad. Stab., 2016, 130, 10-21.

31 H. M. Khanlou, A. Sadollah, B. C. Ang, J. H. Kim, S. Talebian and A. Ghadimi, Prediction and optimization of electrospinning parameters for polymethyl methacrylate nanofiber fabrication using response surface methodology and artificial neural networks, Neural Comput. Appl., 2014, 25(3-4), 767-777.

32 B. T. Pham, M. D. Nguyen, K.-T. T. Bui, I. Prakash, K. Chapi and D. T. Bui, A novel artificial intelligence approach based on multi-layer perceptron neural network and biogeographybased optimization for predicting coefficient of consolidation of soil, Catena, 2019, 173, 302-311.

33 A. Jahani, Modeling of forest canopy density confusion in environmental assessment using artificial neural network, Iranian Journal of Forest and Poplar Research, 2016, 24(2), 310-321.

34 A. Jahani and F. A. Mohammadi, Aesthetic quality modeling of landscape in urban green space using artificial neural network, Journal of Natural Environment, 2017, 69(4), 951963.

35 A. Jahani, Sycamore failure hazard classification model (SFHCM): an environmental decision support system (EDSS) in urban green spaces, Int. J. Environ. Sci. Technol., 2019, 16(2), 955-964.

36 S. Shafiee and S. Minaei, Combined data mining/NIR spectroscopy for purity assessment of lime juice, Infrared Phys. Technol., 2018, 91, 193-199.

37 Q. He, H. Shahabi, A. Shirzadi, S. Li, W. Chen, N. Wang, et al., Landslide spatial modelling using novel bivariate statistical based Naïve Bayes, RBF Classifier, and RBF Network machine learning algorithms, Sci. Total Environ., 2019, 663, 1-15.

38 Y. Yao, L. Dai, F. Jiang, W. Liao, M. Dong and X. Yang, Kinetic modeling of novel solid desiccant based on PVALiCl electrospun nanofibrous membrane, Polym. Test., 2017, 64, 183-193.

39 W. Lü, Y. Chen, W. Ma, X. Zhang, F. Luan, M. Liu, et al., QSAR study of neuraminidase inhibitors based on heuristic method and radial basis function network, Eur. J. Med. Chem., 2008, 43(3), 569-576.

40 N. Qu, X. Li, Y. Dou, H. Mi, Y. Guo and Y. Ren, Nondestructive quantitative analysis of erythromycin ethylsuccinate powder drug via short-wave near-infrared spectroscopy combined with radial basis function neural networks, Eur. J. Pharm. Sci., 2007, 31(3-4), 156-164.

41 L. Wang, Support vector machines: theory and applications, Springer Science \& Business Media, 2005.

42 W.-H. Chen, S.-H. Hsu and H.-P. Shen, Application of SVM and ANN for intrusion detection, Comput. Oper. Res., 2005, 32(10), 2617-2634.

43 A. Jahani, Sycamore failure hazard risk modeling in urban green space, Journal of Spatial Analysis Environmental Hazards, 2017, 3(4), 35-48. 
44 S. Akbarifard and B. Bakhtiari, Optimal allocation of water resources using Water Cycle Algorithm (WCA) (Case study: Gorganrood basin), Water Engineering, 2018, 11(36), 33-46.

45 S. Rezazadeh, A. Jahani, M. Makhdoum and H. G. Meigooni, Evaluation of the Strategic Factors of the Management of Protected Areas Using SWOT Analysis-Case Study: Bashgol Protected Area-Qazvin Province, Open J. Ecol., 2017, 7(01), 55-68.

46 R. Khatibi, M. A. Ghorbani and F. A. Pourhosseini, Stream flow predictions using nature-inspired Firefly Algorithms and a Multiple Model strategy - Directions of innovation towards next generation practices, Adv. Eng. Inf., 2017, 34, 80-89.

47 M. A. Ghorbani, R. C. Deo, Z. M. Yaseen, M. H. Kashani and B. Mohammadi, Pan evaporation prediction using a hybrid multilayer perceptron-firefly algorithm (MLP-FFA) model: case study in North Iran, Theor. Appl. Climatol., 2018, 133(3-4), 1119-1131.

48 S. Kim, Y. Seo, M. Rezaie-Balf, O. Kisi, M. A. Ghorbani and V. P. Singh, Evaluation of daily solar radiation flux using soft computing approaches based on different meteorological information: peninsula $v s$. continent, Theor. Appl. Climatol., 2018, 137(1-2), 1-20.

49 S. Niell, F. Jesús, R. Díaz, Y. Mendoza, G. Notte, E. Santos, et al., Beehives biomonitor pesticides in agroecosystems: Simple chemical and biological indicators evaluation using Support Vector Machines (SVM), Ecol. Indicat., 2018, 91, 149-154.

50 F. Tourlomousis and R. C. Chang, Dimensional metrology of cell-matrix interactions in $3 \mathrm{D}$ microscale fibrous substrates, Procedia CIRP, 2017, 65, 32-37.

51 R. Laref, E. Losson, A. Sava and M. Siadat, On the optimization of the support vector machine regression hyperparameters setting for gas sensors array applications, Chemom. Intell. Lab. Syst., 2019, 184, 22-27.

52 M. M. Abolhasani, K. Shirvanimoghaddam, H. Khayyam, S. M. Moosavi, N. Zohdi and M. Naebe, Towards predicting the piezoelectricity and physiochemical properties of the electrospun $\mathrm{P}(\mathrm{VDF}-\mathrm{TrFE})$ nanogenerators using an artificial neural network, Polym. Test., 2018, 66, 178-188.

53 N. Ketabchi, M. Naghibzadeh, M. Adabi, S. S. Esnaashari and R. Faridi-Majidi, Preparation and optimization of chitosan/ polyethylene oxide nanofiber diameter using artificial neural networks, Neural Comput. Appl., 2017, 28(11), 31313143.

54 T. Khatti, H. Naderi-Manesh and S. M. Kalantar, Application of ANN and RSM techniques for modeling electrospinning process of polycaprolactone, Neural Comput. Appl., 2019, 31(1), 239-248.

55 Neural network modeling of smart nanostructure sensor for electronic nose application, 6th International Conference on
Systems and Control (ICSC), ed. S. Khaldi and Z. Dibi, Batna, Algeria, 2017.

56 E. Hosaini-Alvand, H. Mirshekar, M. Taghi Khorasani, M. Parvazinia and A. Joorabloo, Fabricating and robust artificial neural network modeling nanoscale polyurethane fiber using electrospinning method, J. Appl. Polym. Sci., 2017, 134(30), 45116.

57 S. Baghersad, S. H. Bahrami, M. R. Mohammadi, M. R. M. Mojtahedi and P. B. Milan, Development of biodegradable electrospun gelatin/aloe-vera/poly( $\varepsilon$ caprolactone) hybrid nanofibrous scaffold for application as skin substitutes, Mater. Sci. Eng. C, 2018, 93, 367-379.

58 D. Nurwaha, W. Han and X. Wang, Effects of processing parameters on electrospun fiber morphology, J. Text. Inst., 2013, 104(4), 419-425.

59 C. Thompson, G. G. Chase, A. Yarin and D. Reneker, Effects of parameters on nanofiber diameter determined from electrospinning model, Polymer, 2007, 48(23), 6913-6922.

$60 \mathrm{~S}$. Theron, E. Zussman and A. Yarin, Experimental investigation of the governing parameters in the electrospinning of polymer solutions, Polymer, 2004, 45(6), 2017-2030.

61 A. Haghi, Electrospun nanofibers research: Recent developments, Nova Science Publishers Incorporated, 2009.

62 M. Costolo, J. Lennhoff, R. Pawle, E. Rietman and A. Stevens, A nonlinear system model for electrospinning sub-100 nm polyacrylonitrile fibres, Nanotechnology, 2007, 19(3), 035707.

63 Q. Shao, R. C. Rowe and P. York, Comparison of neurofuzzy logic and neural networks in modelling experimental data of an immediate release tablet formulation, Eur. J. Pharm. Sci., 2006, 28(5), 394-404.

64 S. Ramakrishna, An introduction to electrospinning and nanofibers, World Scientific, 2005.

65 T. Wu, X. Chen, J. Sha, Y.-Y. Peng, Y.-L. Ma, L.-S. Xie, et al., Fabrication of shish-kebab-structured carbon nanotube/ poly( $\varepsilon$-caprolactone) composite nanofibers for potential tissue engineering applications, Rare Met., 2019, 38(1), 6472.

66 G. G. Ahmad, Using artificial neural networks with graphical user interface to predict the strength of carded cotton yarns, J. Text. Inst., 2016, 107(3), 386-394.

67 F. A. Paskiabi, E. Mirzaei, A. Amani, M. A. Shokrgozar, R. Saber and R. Faridi-Majidi, Optimizing parameters on alignment of PCL/PGA nanofibrous scaffold: An artificial neural networks approach, Int. J. Biol. Macromol., 2015, 81, 1089-1097.

68 S. S. Esnaashari, M. Naghibzadeh, M. Adabi and R. Faridi Majidi, Evaluation of the Effective Electrospinning Parameters Controlling Kefiran Nanofibers Diameter Using Modelling Artificial Neural Networks, Nanomed. Res. J., 2017, 2(4), 2239-2349. 